\title{
Characteristics of Helium Gas with High Temperature and High Pressure Flowing through a 90-Degree Elbow
}

\author{
Beibei Feng, ${ }^{1}$ Yanfei Sun, ${ }^{1}$ Xingtuan Yang, ${ }^{1}$ Shengqiang Li, ${ }^{1}$ \\ Jiyuan Tu, ${ }^{1,2}$ and Shengyao Jiang ${ }^{1}$ \\ ${ }^{1}$ Key Laboratory of Advanced Reactor Engineering and Safety, Ministry of Education, Institute of Nuclear and New Energy Technology, \\ Tsinghua University, Beijing 100084, China \\ ${ }^{2}$ School of Aerospace, Mechanical \& Manufacturing Engineering, RMIT University, Plenty Road, Bundoora, VIC 3083, Australia
}

Correspondence should be addressed to Beibei Feng; fengbeibei@tsinghua.edu.cn

Received 27 November 2013; Accepted 6 January 2014; Published 14 April 2014

Academic Editors: A. R. Beig and H. Zhang

Copyright (C) 2014 Beibei Feng et al. This is an open access article distributed under the Creative Commons Attribution License, which permits unrestricted use, distribution, and reproduction in any medium, provided the original work is properly cited.

\begin{abstract}
There exists a certain $90^{\circ}$ elbow structures in the helium circulation of HTGR-10. In terms of energy-saving and design simplification of reactor's primary loop, $90^{\circ}$ elbow can be used to measure the helium flow and the content of water vapor, both of which are significant in an accident. It is necessary to make an in-depth research of the flow characteristics of helium flowing $90^{\circ}$ elbow. Simulation results indicate that fluid's motion in the elbow is under the control of the centrifugal forces. Static pressure near the extrados is higher than that near the intrados. Boundary layer separation occurs at the latter half intrados of the elbow. The vortex emerges during the separation process and increases the energy dissipation. Velocity in the near-intrados region is higher than that in the near-extrados region, which is opposite to the pressure distribution trend. Under the action of the centrifugal forces, the secondary flow emerges in the latter half of the elbow and complicates the flow field by generating two vortexes which rotate in a different direction.
\end{abstract}

\section{Introduction}

High temperature gas-cooled reactor (HTGR), which is developed from gas-cooled reactor and advanced gas-cooled reactor, is an advanced type of reactor in nuclear power reactors. High temperature gas-cooled reactor has a lot of advantages over traditional rectors, such as higher inherent safety, better economy, and higher power generation efficiency, and also the process heat generated by HTGR can be applied in nuclear hydrogen production [1] and some other fields. The HTGR has the potential to become one of the advanced reactors which will be upgraded preferentially [2]. The primary circuit of high temperature gas-cooled reactor works with helium as the cooling medium. The helium flow through the gap between the spherical fuel elements takes away the heat generated by the fission of U-235 and then transfers the heat to the steam generator. Then, the working fluid of the secondary circuit absorbs the heat energy and moves the steam turbine work. The system figure of a 10-MW HTGR is shown in Figure 1.

The integrality and resistance characteristic of the primary circuit has a significant influence on the safety and economy of HTGR. The accurate measurement of the helium's flow and pressure is the precondition for safe and stable operation of the HTGR, especially in an accident. At present, these critical parameters are measured by additional local resistance component. But additional local resistance components engender some serious harm that goes against the safety and economy. For example, additional local resistance components make the force conditions of the circuit pipe more complicated, which add additional difficulties to the design of the primary circuit. On the other hand, additional local resistance components increase the resistance of the primary circuit. This makes the helium circulator consume more power to maintain the forced circulation of helium, and this is bad for the economy. Considering that 


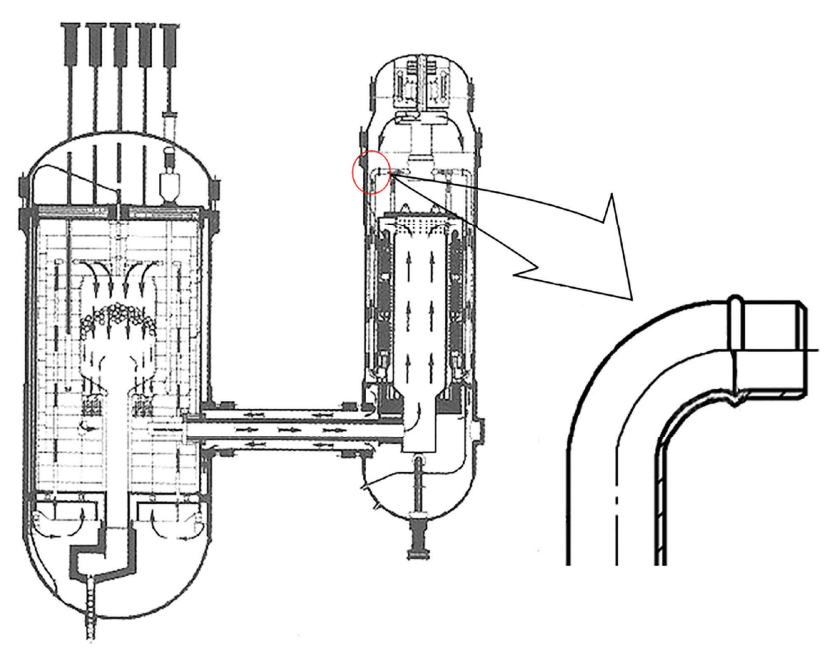

FIGURE 1: The primary circuit of the HTGR-10.

there inevitably exist some elbow structures in the primary circuit, as shown in Figure 1, we can make use of the elbow as the sensing element to monitor the fluctuation of the helium's flow and pressure, even to measure the steam content mixed in the helium. The advantage of doing this is simplifying the force conditions of the primary circuit and decreasing the resistance, by which the design of the primary circuit is less difficult than before and the economy is improved.

In order to apply the bend sensor to measure helium's parameter in the HTGR's helium circulation loop, the flow characteristic of helium in an elbow under the condition of high temperature and high pressure must be analyzed profoundly and in detail to research the nature of helium flow in an elbow. Liangying and Zhengrong $[3,4]$ analysed the influence of elbow parameters on flow measurement and discussed the practicability and the reliability of elbow flowmeter. Also the PHOENICS software has been used to simulate the pressure distribution by Liangying and Zhengrong, and the relationship between the flow and the maximum pressure difference is in accord with the results of experiments. From the viewpoint of improving the measuring method by using elbow flowmeter, Sun et al. [5] researched the relationship between the stability of pressure measurement value and pressure measuring point. Sun's results showed that when the pressure measuring point is located at the position which constitutes an angle of 45 degrees with the outlet crosssection, the sensitivity of pressure difference measurement will be improved and the influence of the secondary flow on measurement correspondingly is reduced. Guomin and Yi [6] analyzed the feasibility of the use of elbow flowmeter in multiphase flow measurement and design some kinds of measuring method by combining the elbow flowmeter and densimeter. Zhi et al. [7] put forward a swirling flow theory model based on N-S equation numerical solution, which made an improvement on the theoretical research of elbow flowmeter based on traditional free swirl theory and forced swirl theory. But the disadvantage of this theory is that a large number of differential equations have to be solved with

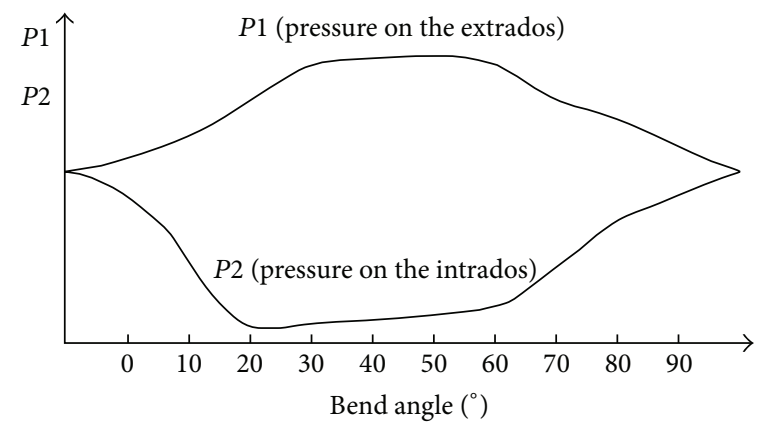

FIGURE 2: The pressure measurement results on different test point.

the help of the computer before we get an accurate flow coefficient. Enayet et al. [8] observed the separation of the boundary layer at the outlet of the elbow experimentally. The results are in good agreement with the known flow phenomenon in an elbow. Kimura et al. [9] researched the influence of the elbow on the downstream flow. And the close relationship between the velocity fluctuation intensity and the secondary flow also has been found. Boiron et al. [10] researched the influence of inlet conditions on the secondary flow experimentally and numerically.

According to these theoretical results, a large number of researchers have conducted experiments $[5,6]$ to research the use of bend sensor in the flow measurement field. First of all, the purpose is to determine the best pressure measuring point in order to improve the measurement precision. The experiment results are shown in Figure 2.

We can see that when the pressure measuring point is located between $35^{\circ}$ and $55^{\circ}$ on the extrados or intrados of the elbow, the observed value of static pressure is almost a constant. This is the foundation of choosing a couple of perfect pressure measuring points, of which the advantage is that the pressure difference between the two points is stable and large, both of which are good for measurement.

It is feasible to apply the bend sensor in the primary circuit of HTGR according to the discussion above. But more information relating to the helium flow in an elbow must be learned first. Our research is concentrated on the flow details of helium in the elbow and analyzes the pressure and velocity distribution.

\section{Numerical Model}

In order to study the flow characteristics of helium in the elbow, the computational model consisted of a horizontal inlet pipe with a length of 2 pipe diameters, a 90-degree horizontal-placed elbow with a bending diameter ratio of 1.5 , and a horizontal outlet pipe with a length of 6 pipe diameters as seen in Figure 3. The detailed geometry parameter is shown in Table 1.

The RANS simulation with $k$-epsilon model is performed with "FLUENT" computational fluid dynamics software. Standard $k$-epsilon turbulence model is used to model turbulent helium or helium-vapor flows through the elbow due 


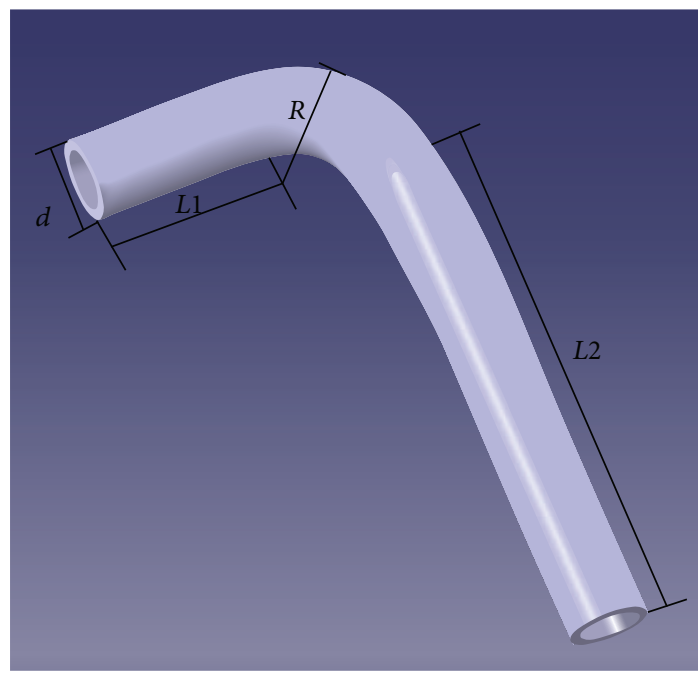

FIGURE 3: The geometry model of the elbow structure.

TABLE 1: The geometry parameter of the structure model.

\begin{tabular}{|c|c|c|c|c|}
\hline $\begin{array}{l}\text { Pipe } \\
\text { diameter/mm }\end{array}$ & $\begin{array}{c}\text { Radius of } \\
\text { curvature/mm }\end{array}$ & $\beta=R / d$ & $\begin{array}{l}\text { Inlet pipe } \\
\text { length } \\
(L 1)\end{array}$ & $\begin{array}{l}\text { Outlet } \\
\text { pipe } \\
\text { length } \\
(L 2)\end{array}$ \\
\hline 233 & 349.5 & 1.5 & 349.5 & 1048.5 \\
\hline
\end{tabular}

TABLE 2: The results of three refinement solutions.

\begin{tabular}{lccc}
\hline & \multirow{2}{*}{ Cell number } & \multicolumn{2}{c}{ Dynamic pressure/Pa } \\
& & Min. & Max. \\
\hline A & 61440 & 230 & 4023 \\
B & 344064 & 242 & 4072 \\
C & 442368 & 245 & 4093 \\
Error & B to A & $5.6 \%$ & $1.2 \%$ \\
& C to B & $1.2 \%$ & $0.5 \%$ \\
\hline
\end{tabular}

to its widespread utilization and less consumption and also good accuracy.

Fully developed turbulent flow is assumed in the horizontal inlet section, and after the elbow, the long outlet pipe can guarantee that the flow of helium will recover to the initial state. A "no-slip" boundary condition is employed for the helium velocity at the wall surface and the thermal conditions are set in thermal isolation.

In order to achieve a good prediction of the flow field, hexahedral mesh has been used to disperse the computational domain. The mesh has been refined for many times to eliminate the influence of mesh quality in order to increase the computational accuracy. The three computational results of the refinement solution are shown in Table 2.

Table 2 tells us that when the cell number of the computational domain changes from 61400 to 344064 , the dynamic pressure in the near-wall region makes a considerable change. While when it changes from 34406 to 442368 , the magnitude of the relative error between the two simulation results is less

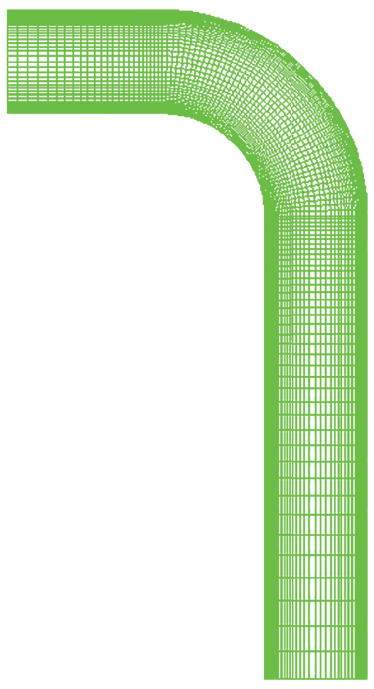

(a) Original mesh

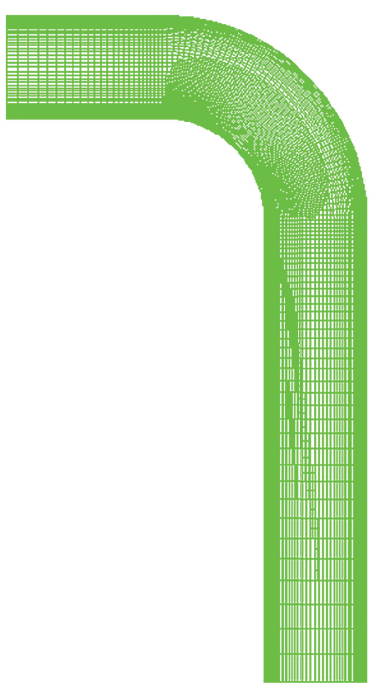

(b) Final mesh

FIGURE 4: The meshing condition of the geometry model.

than $2 \%$. Considering the time-consuming and the acceptable computational accuracy, the refinement solution of 344064 cell number is applied in our simulation.

Considering that the pressure gradient varies significantly in the elbow, we refine the mesh again to adapt it to the pressure gradient which is based on the results of preliminary calculations. 10 percent of the maximum of the pressure gradient is set as the refined threshold. Then the original and final mesh of the symmetry plane both are shown in Figure 4, from which we can see that the nearer to the wall, the finer the meshes.

The helium has a mass flow of $6.86 \mathrm{~kg} / \mathrm{s}$ which is set as the inlet boundary condition. The temperature is 250 centigrade and the global pressure is set as $7.0 \times 10^{6} \mathrm{~Pa}$. Under this condition, the density of helium is $6.442 \mathrm{~kg} / \mathrm{m}^{3}$ and the dynamic viscosity is $2.886 \times 10^{-5} \mathrm{~kg} /(\mathrm{m} \cdot \mathrm{s})$. Standard 


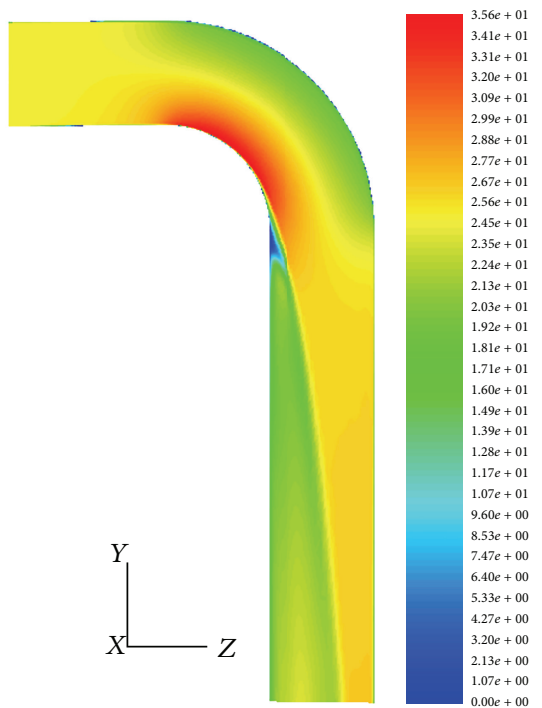

FIGURE 5: Velocity contour of helium in the symmetry plane.

wall functions have been used in the simulation and the $y$ plus of the near-wall region is between 48.7 and 264.6 under the condition of the final mesh. Reynolds number is about $1.3 \times 10^{6}$, and Dean number is about $7.5 \times 10^{5}$, both of which are much larger than that presented in the references.

\section{Results and Discussion}

3.1. The Velocity Distribution in the Elbow. Figure 5 shows the velocity contour of helium in the symmetry plane. The magnitude of the velocity is shown by color. The high velocity region is observed at the intrados of the elbow in the figure, and the low velocity region is at the extrados. Boundary layer separation occurred at the latter half intrados of the elbow. To be more precise, the separation point exists at the location just a little before the elbow outlet (line B). These results are qualitatively in good agreement with well-known flow behavior in the elbow (Enayet et al., 1982) [8].

Figures 6 and 7 show the experimental results of Kimura et al. [9]. Figure 6 shows the streamline of time averaged velocity in the cross-section along the center axis of the elbow. The color stands for the magnitude of the velocity which has been normalized by the average velocity in the inlet pipe.

Figure 7 shows the time averaged velocity vectors perpendicular to the pipe axis at the elbow outlet in the circular cross-section. Near the intrados of the pipe (lower side in the figure), the high velocity region is observed. In the circular cross-section, the fluid had a component velocity of which the direction is from the intrados to the extrados of the elbow. The flow corresponds to the high velocity region shown in Figure 6. This flow with high velocity, due to the action of centrifugal forces, originated a biased velocity distribution in the latter pipe. Furthermore, circumferential flows along the pipe wall are also observed. These flows resulted in the secondary flow and biased velocity distribution in the latter pipe. The secondary flow and biased velocity distribution can affect the flow regime of the latter pipe flow.

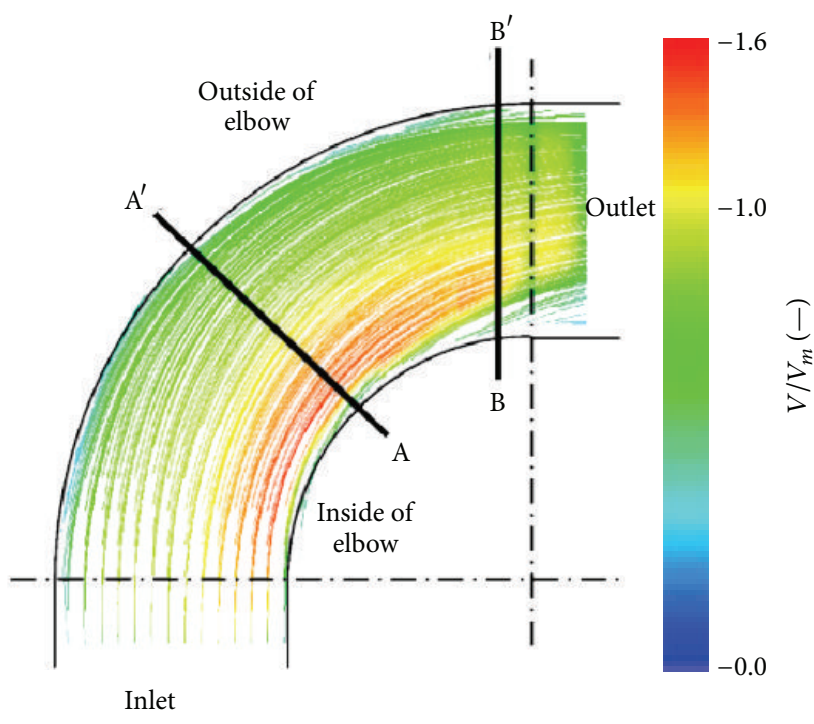

FIgURE 6: Time averaged streamline in elbow.

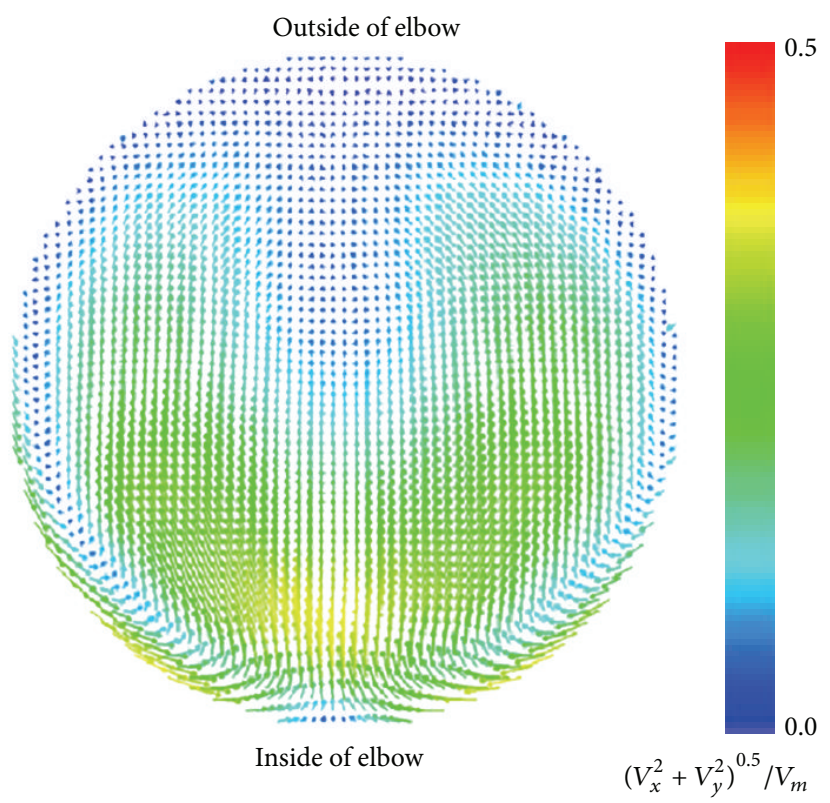

FIgURE 7: Time averaged velocity vectors at elbow outlet in circular cross-section.

3.2. The Pressure Distribution in the Elbow. Figure 8 shows the static pressure distribution in the domain. Before the elbow, the flow is stable and the static pressure is a constant for all of the cross-section. When it comes to the elbow, the helium does a quarter of circular motion due to the restraint of the elbow's inner wall. Hence, the fluid's motion in the elbow is also under the control of the centrifugal forces.

The centrifugal forces are given by the pressure difference between two points which are the same in bend radius direction but different in bend radius value. So, we can infer that static pressure near the extrados is higher than that near the intrados. The simulation results in Figure 8 rigorously confirm our inference. Along the bend radius direction, 


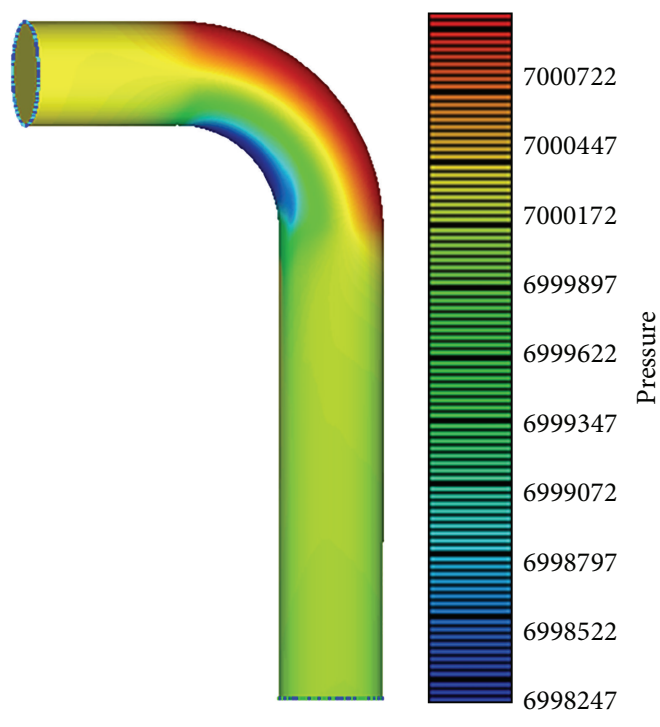

FIGURE 8: Pressure contours in the elbow.

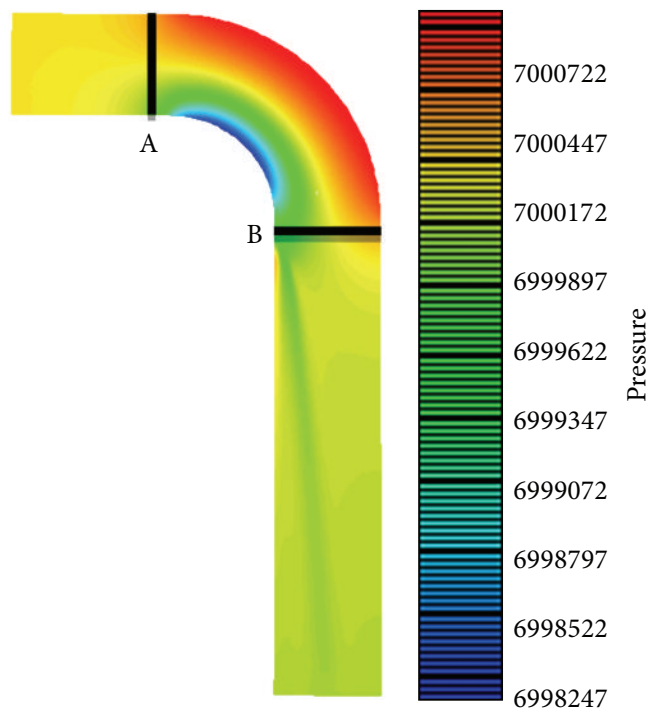

Figure 9: Pressure contours on the symmetry plane.

the static pressure becomes higher with the increase of the radius value. The maximum of local static pressure is about $7000945 \mathrm{~Pa}$ which is located at the extrados of the elbow and the minimum is about $6998247 \mathrm{~Pa}$, located at the intrados. The difference between the two values reaches $2700 \mathrm{~Pa}$.

Figure 9 shows the static pressure distribution in the symmetry plane of the domain. We can find that, from crosssection A to cross-section $\mathrm{B}$, the color of the static pressure contour varies with the value of bend radius and the biggest fluctuation of static pressure distribution also exists in this region because the color difference of the pressure contour, which stands for pressure difference, is at its maximum. As the influence of the elbow, the starting point of flow field transformation of helium emerges before the elbow inlet, namely, cross-section A, as shown in Figure 9. And after the elbow outlet, namely, cross-section $\mathrm{B}$, the static pressure

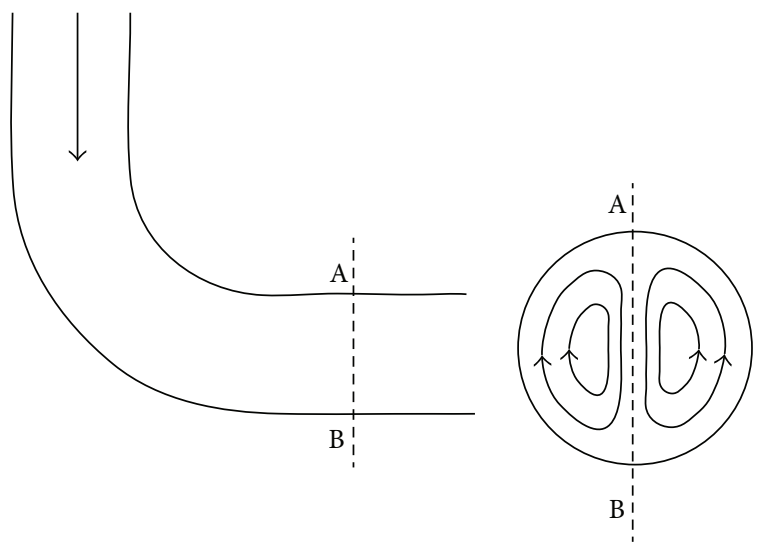

Figure 10: The forming process of the second flow.

begins to recover to a relatively stable state gradually. But, due to the energy dissipation induced by viscous forces and local resistance of elbow, the static pressure cannot recover to its original state. So, the color near the outlet of the pipe is lighter than that of the inlet which indicates that the static pressure has decreased irreversibly.

3.3. The Secondary Flow in the Elbow. According to the simulation, the secondary flow has been observed. The forming process of the secondary flow can be illustrated by the transverse velocity gradient. The process is shown in Figure 10. A stands for intrados and B represents extrados.

When fluid flows in an elbow, as we know, velocity field near the intrados is stronger than that near the extrados. When the fluid does a circular motion, effects on nearintrados fluid made by centrifugal forces are more significant than those on near-extrados. Then the core fluid will flow from the near-intrados region to the near-extrados region under the driving of the centrifugal forces. In the nearextrados region, the pressure is higher than that of the nearintrados region. Hence, under the driving of the pressure difference, the fluid will flow back from the near-extrados region to the near-intrados region. Then the secondary flow occurs and exists in the elbow flow or some other curvedpipe flow. Because of this, in the elbow pipe flow, the main flow along the axis will be overlaid by a secondary flow which is perpendicular to the axis. In the center of the pipe, the secondary flow direction is from the nearintrados region to the near-extrados region, and in the nearwall region, the direction is contrary to the former. The secondary flow complicates the flow field by generating two vortexes which rotate in a different direction. Velocity on the circular cross-section fluctuated greatly due to the secondary flow. The elbow can cause biased velocity distribution and large velocity fluctuation with low frequency. The formation of the secondary flow needs two foundation conditions, namely, the centrifugal forces field and the shear stress gradient. In the elbow pipe flow, the formation conditions are automatically satisfied. So, the pressure field we measure has been influenced by the secondary flow. In consideration of the significance of accuracy in measurement of nuclear 
engineering, the influence of the secondary flow must be taken into account.

3.4. Boundary Layer Separation at the Latter Half Intrados of the Elbow. Boundary layer is a thin layer close to object surface, in which there exist a very large velocity gradient and rotation along the normal direction. Boundary layer separation has been observed at the latter half intrados of the elbow. The separation process can be described as follows: when fluid flows through a curved surface, along the flow direction, the flow field near the wall can be divided into three regions, namely, favorable pressure gradient region, zero-pressuregradient region, and the adverse pressure gradient region. In favorable pressure gradient region, the fluid element near the wall goes forward by the driving of the pressure difference. Then, when it goes into the zero-pressure-gradient region, the fluid element overcomes the viscous drag by consuming its own kinetic energy and continues to flow forward. And then the fluid element ingresses the adverse pressure gradient, in which the element will decelerate under the action of viscous force and adverse pressure difference. The kinetic energy will be consumed up and the velocity turns to be zero. Then the element will depart from the wall and the space will be filled by the retral fluid. Vortex emerges during the separation process and increases the energy dissipation. Also the pressure field in the elbow is disturbed. The boundary layer separation has a great influence on the flow field, especially on the downstream flow field. So if the elbow sensor is applied in the measurement system of HTGR-10, the boundary layer separation process must be researched profoundly.

\section{Conclusions}

An investigation of the flow characteristics of helium flows after a 90-degree horizontal elbow under conditions of $7.0 \times$ $10^{6} \mathrm{~Pa}$ and 250 centigrade is carried out numerically by using the standard $k$-epsilon turbulence model. The simulation results show that the fluid's motion in the elbow is under the control of the centrifugal forces. The static pressure near the extrados is higher than that near the intrados. Along the bend radius direction, the static pressure becomes higher with the increase of the radius value. When the pressure measuring point is located between $35^{\circ}$ and $55^{\circ}$ on the extrados or intrados, the observed value of static pressure is almost a constant. Boundary layer separation occurs at the latter half intrados of the elbow and has a great influence on the downstream flow field. Vortexes emerge during the separation process and increase the energy dissipation. The velocity in the near-intrados region is higher than that in the near-extrados region, which is opposite to the pressure distribution trend. Under the action of the centrifugal forces, the secondary flow emerges in the latter half of the elbow and complicates the flow field by generating two vortexes which rotate in a different direction. Velocity on the circular cross-section fluctuated greatly due to the secondary flow. The elbow can cause biased velocity distribution and large velocity fluctuation with low frequency.
So, the elbow sensor can be applied to measure helium's parameter in a HTGR according to the research above. But we must make an in-depth research on the flow characteristics of helium in an elbow. Further study is needed to search the nature of helium flow in an elbow. Considering that there may be a little water vapor existing in helium, the flow characteristics of the mixture through the elbow should also be investigated from the viewpoint of two-phase flow to analyze the influence of the water vapor content on the flow coefficients and the pressure fluctuation at test point.

\section{Conflict of Interests}

The authors declare that they have no conflict of interests regarding the publication of this paper.

\section{Acknowledgments}

This project is supported by the National Science and Technology Major Project (Grant no. ZX06901) and The Tribology Science Fund of State Key Laboratory of Tribology (Grant no. SKLTKF12B16).

\section{References}

[1] P. Zhang, Y. Bo, J. Chen, and J. Xu, "Nuclear hydrogen production and high temperature gas-cooled rector," Journal of Chemical Industry and Engineering, vol. 55, 2004.

[2] F. Xiaoming and W. Jie, “The development of high temperature gas-cooled reactor in China," Modern Electric Power, vol. 23, no. 5, pp. 70-75, 2006.

[3] W. Liangying and Z. Zhengrong, "Characteristics of curved pipe and their applications in the flow measurement," Industrial Heating, vol. 4, pp. 39-41, 2000.

[4] W. Liangying and Z. Zhengrong, "The theoretical analysis and experimental research of the application of elbow flowmeter in the flow measurement," Measurement Technique, vol. 9, pp. 3234, 2003.

[5] Z.-Q. Sun, J.-M. Zhou, and H.-J. Zhang, "Numerical simulation and experimental research on measurement characteristics of elbow meter," Chinese Journal of Sensors and Actuators, vol. 20, no. 6, pp. 1412-1415, 2007.

[6] X. Guomin and S. Yi, "The experimental research of the application of elbow flowmeter in the measurement of multiphase flow," Oil-Gasfield Surface Engineering, vol. 25, no. 10, pp. 10-11, 2006.

[7] L. Zhi, M. Xianju, and L. Shaofeng, "Theoretic research on elbow ducts based on the N-S equation," Journal of Hebei Polytechnic University, vol. 30, no. 1, pp. 41-45, 2008.

[8] M. M. Enayet, M. M. Gibson, A. M. K. P. Taylor, and M. Yianneskis, "Laser-Doppler measurements of laminar and turbulent flow in a pipe bend," International Journal of Heat and Fluid Flow, vol. 3, no. 4, pp. 213-219, 1982.

[9] N. Kimura, H. Ogawa, and H. Kamide, "Experimental study on fluid mixing phenomena in T-pipe junction with upstream elbow," Nuclear Engineering and Design, vol. 240, no. 10, pp. 3055-3066, 2010.

[10] O. Boiron, V. Deplano, and R. Pelissier, "Experimental and numerical studies on the starting effect on the secondary flow in a bend," Journal of Fluid Mechanics, vol. 574, pp. 109-129, 2007. 

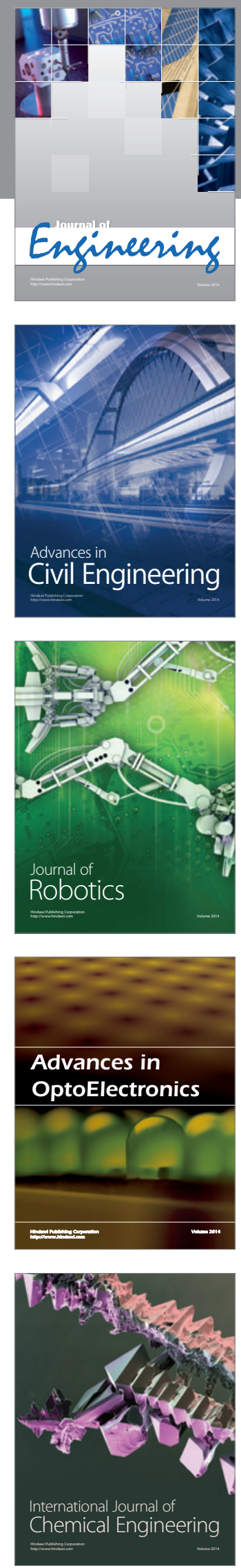

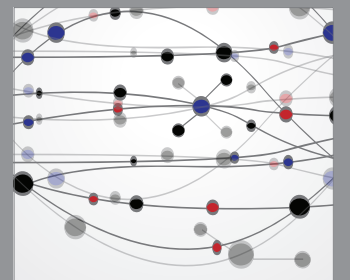

The Scientific World Journal
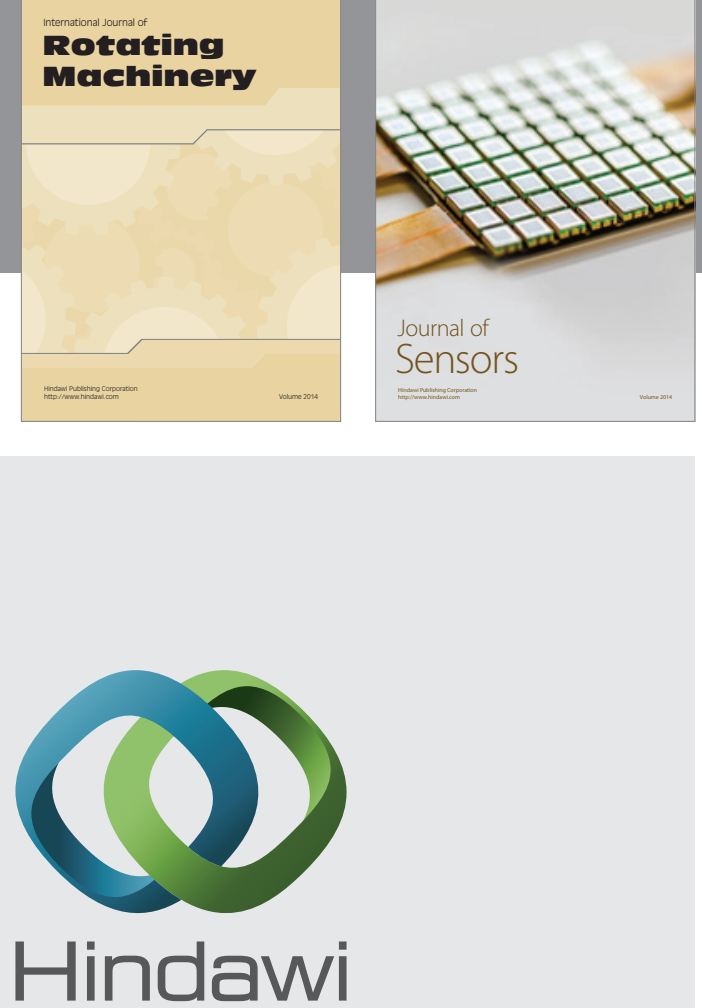

Submit your manuscripts at http://www.hindawi.com
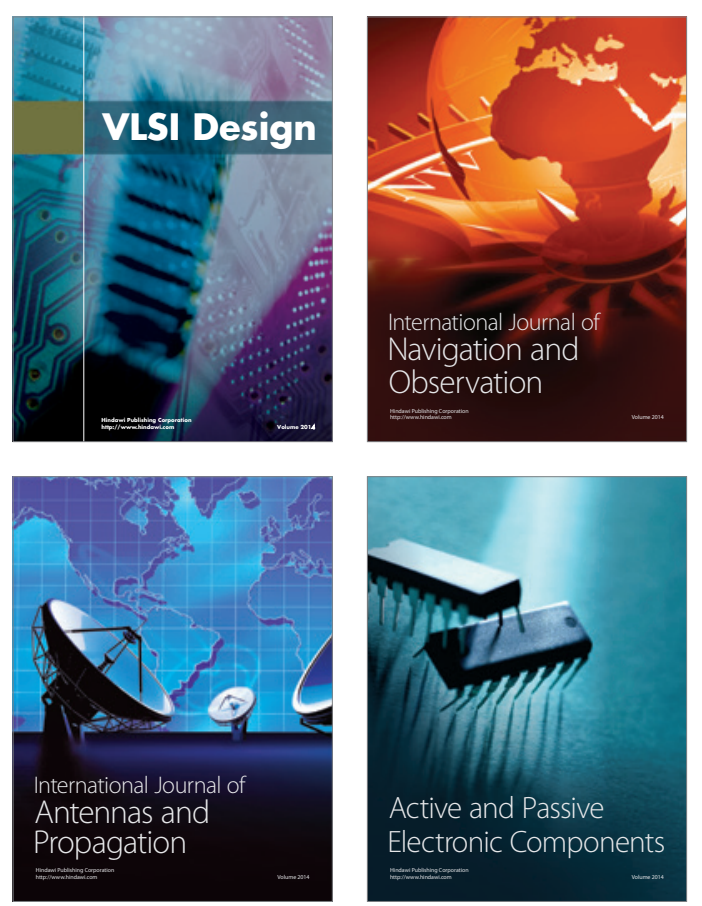
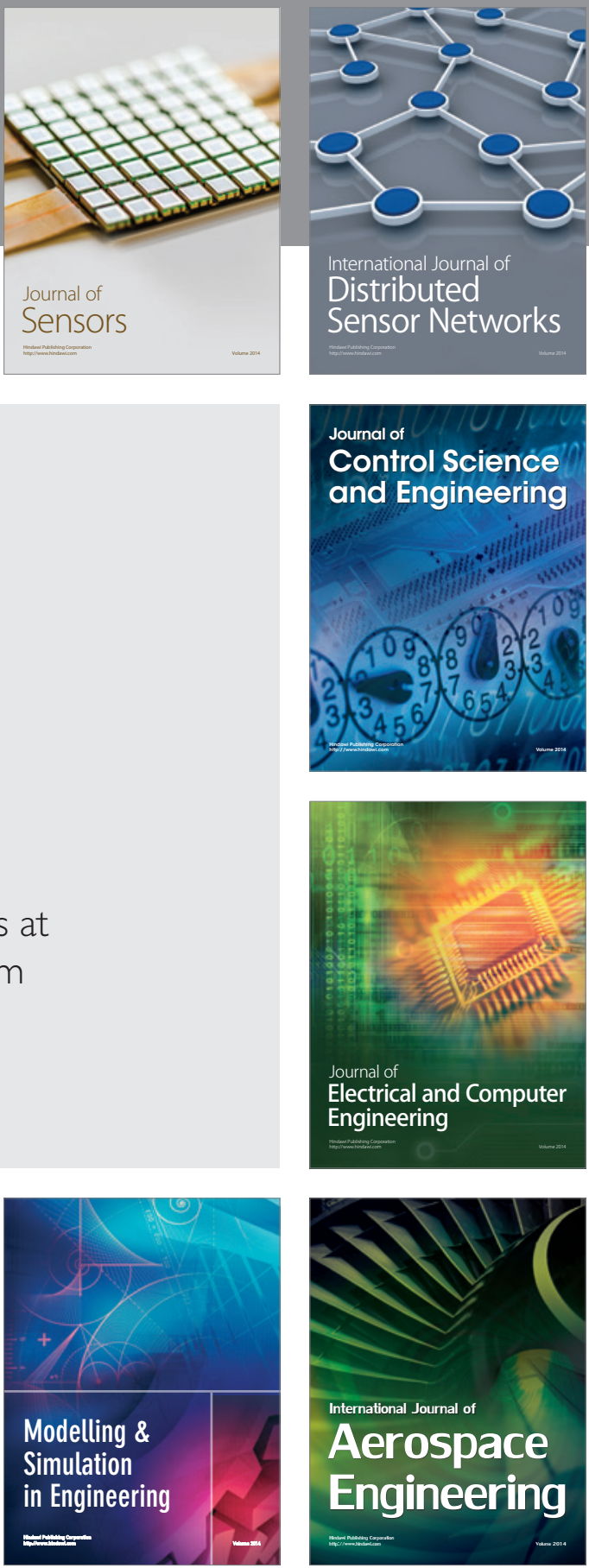

Journal of

Control Science

and Engineering
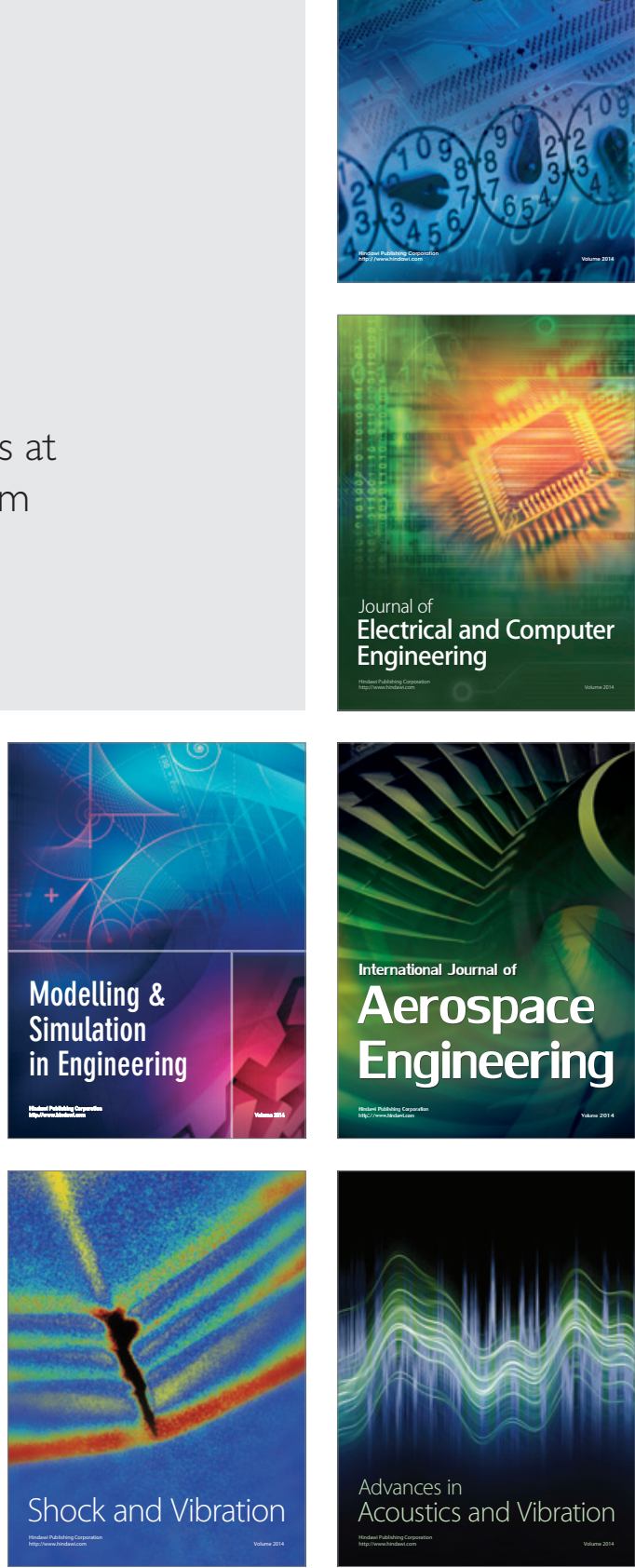